\title{
Zikloteka, centro de documentación de la bicicleta. Una iniciativa avanzada de recopilación, tratamiento y difusión de documentación sobre la movilidad ciclista
}

\author{
Zikloteka, a documentation centre in cycle mobility. An advanced \\ initiative to collect, process and disseminate documentation on \\ cycling mobility
}

\author{
Edorta Bergua Jiménez y y Manu González Baragaña² \\ Fecha de recepción: 07-01-2020 - Fecha de aceptación: 23-07-2020 \\ Hábitat y Sociedad (ISSN 2173-125X), n. ${ }^{\circ}$ 13, noviembre de 2020, pp. 265-xxx. \\ http://dx.doi.org/10.12795/HabitatySociedad.2020.i13.15
}

\section{Summary}

During the last two decades, a significant volume of materials related to everyday cycling have been produced in Gipuzkoa. This documentation, scattered in many locations until now, represents a considerable heritage of administrative, scientific, technical and citizens' knowledge produced in this area, as well as underlining the effort undertaken to normalize the use of the bicycle as a means of transport in this territory.

The Gipuzkoa Provincial Council and the Cristina Enea Foundation set up the Zikloteka in 2015, a specialized documentation centre in cycle mobility in Gipuzkoa, in order to identify, compile, preserve and disseminate this heritage, which currently contains over 3,800 references.

The article presents the main characteristics, services, management system and takes stock of its trajectory. The glossary drawn up to classify the materials is its main contribution in the technical documentation area specialized in mobility.

It underlines the collaboration with different entities, such as the Red de Ciudades por la Bicicleta (Cities for Cycling Network), ConBici, and the Network of Environmental Information and Documentation Centres which, complemented by a lengthy search, collection and management process, make it a unique specialized documentation centre in cycle mobility in Europe.

\section{Key words}

Bicycle; Cyclist; Mobility; Document management; Sustainability

\section{Resumen}

En las últimas dos décadas en Gipuzkoa se ha ido generando un importante volumen de materiales relacionados con el ciclismo cotidiano. Esta documentación, hasta ahora dispersa en múltiples ubicaciones, representa un notable patrimonio del conocimiento administrativo, científico, técnico y ciudadano producido en este área, además de poner de manifiesto el esfuerzo realizado para normalizar el uso de la bicicleta como medio de desplazamiento en este territorio.

La Diputación Foral de Gipuzkoa y la Fundación Cristina Enea constituyeron en 2015 la Zikloteka, un centro de documentación especializado en la movilidad ciclista en Gipuzkoa, con el objetivo de identificar, recopilar, custodiar y difundir este patrimonio, que actualmente alberga más de 3800 referencias.

A lo largo del artículo se presentan sus principales características, servicios, sistema de gestión y un balance de su trayectoria. El glosario elaborado para clasificar los materiales constituye su principal aportación al área de documentación técnica especializada en movilidad.

Se subraya la colaboración establecida con distintas entidades, como la Red de Ciudades por la Bicicleta, ConBici, y la Red de Centros de Información y Documentación Ambiental que, acompañada de un largo proceso de búsqueda, acopio y gestión, le configuran como un centro documental especializado en movilidad ciclista único en Europa.

\section{Pallabras clave}

Bicicleta; Ciclista; Movilidad; Gestión documental; Sostenibilidad

1 Técnico urbanista, licenciado en Ciencias Políticas y Sociología. Trabaja como técnico superior de Planificación y Promoción de

Vías Ciclistas en la Diputación Foral de Gipuzkoa. E-mail: edorta@protonmail.com. ORCID: 0000-0002-1756-8589

2 Director técnico del Observatorio de la Sostenibilidad y Clima. Fundación Cristina Enea, Donostia, España. 


\section{Introducción}

La presencia de la bicicleta como medio de desplazamiento en nuestras localidades es un hecho palpable y en continuo aumento. Las dinámicas sociales a favor del ciclismo cotidiano que han ido surgiendo en los últimos 25 años en Gipuzkoa, así como las intervenciones llevadas a cabo en esta materia por parte de las administraciones públicas y la gran acogida ciudadana suscitada han propiciado este hecho.

Como consecuencia de ello, la movilidad ciclista hace tiempo que empezó a incorporarse a la agenda de cada vez más administraciones locales. Al mismo tiempo se configura como un ámbito de creciente interés para distintos actores económicos, y las áreas de investigación científica, desarrollo tecnológico e innovación.

Este proceso ha conllevado una estimable producción de trabajos, estudios y materiales relacionados con esta temática, elaborados por agentes diversos y desde distintas disciplinas. De esta forma se va progresivamente generando en la esfera administrativa, técnica y ciudadana un patrimonio notable del conocimiento ciclista que, sin embargo, se halla desperdigado en infinidad de entidades.

Esta nueva cultura ciclista, institucional, académica, económica y social que emerge está originando intercambios de experiencias, así como una demanda de acceso a fuentes de información especializadas. En efecto, se viene constatando un incremento del número de personas y entidades interesadas en acceder a documentación relacionada con la bicicleta en su vertiente de medio de desplazamiento. Además, en Gipuzkoa el uso ciclista tiene un importante arraigo en su dimensión deportiva y de ocio, ya sea en su versión clásica de ciclismo en carretera, como en su modalidad cicloturista o de paseo, campos todos ellos objeto de amplia producción documental. También se detecta un interés por dar cuenta de la experiencia de la industria ciclista guipuzcoana, en tanto que constituye uno de los pilares de nuestra comunidad e identidad colectiva.

Es en este contexto que en 2015 la Diputación Foral de Gipuzkoa y Fundación Cristina Enea, compartiendo un criterio de responsabilidad pública y de compromiso con la movilidad sostenible,

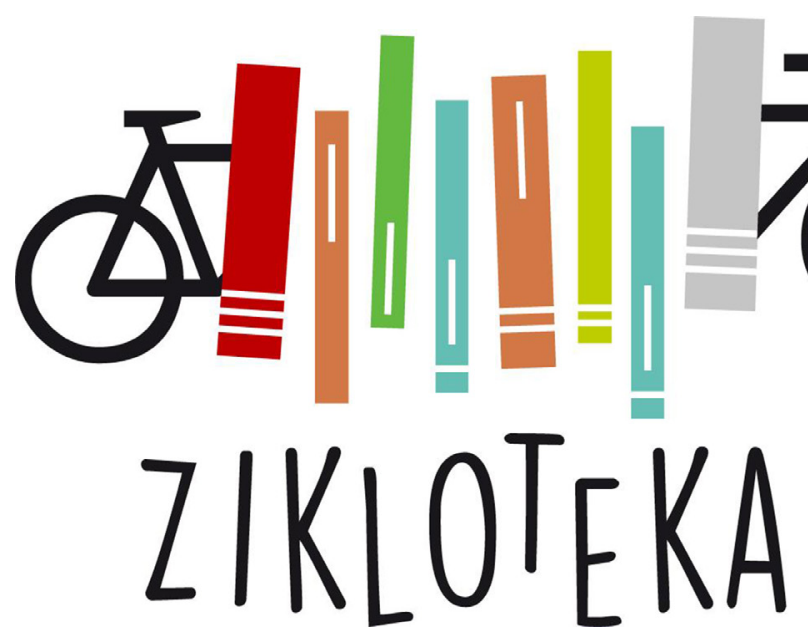

Figura 1. Logotipo de la Zikloteka. Fuente: Zikloteka. Centro de Documentación de la Bicicleta de Gipuzkoa. coinciden en considerar la necesidad de garantizar que las bases documentales que recogen el conocimiento administrativo, técnico y ciudadano acumulado en este ámbito, sean objeto de recopilación, procesamiento y custodia, para posteriormente ponerlas a disposición de profesionales, instituciones, asociaciones y público interesado.

Fruto de ello ese mismo año suscribieron un protocolo de colaboración para crear un centro de documentación especializado en el área de la movilidad ciclista de Gipuzkoa, al que se denominó «Zikloteka» (ver logo oficial en Figura 1).

Se trata de un espacio físico y virtual, que clasifica, archiva, difunde y facilita el acceso a la documentación escrita, gráfica y audiovisual que recopila en sus fondos.

Tras 4 años de rodadura Zikloteka se ha configurado como un centro público documental especializado en la movilidad ciclista que es único en Europa y referencia internacional. Prueba de ello es que el Plan Estratégico Estatal de la Bicicleta, liderado por la Dirección General de Tráfico, lo ha seleccionado como práctica de referencia y 
que, como más adelante se expondrá, cuenta con el apoyo de los principales actores que operan en este ámbito en el Estado español.

\section{Servicios que oferta la Zikloteka}

La Zikloteka abrió sus puertas al público a comienzos de 2016, en su sede ubicada en el Centro de Recursos Medio Ambientales de Fundación Cristina Enea, en San Sebastián (ver Figura 2).

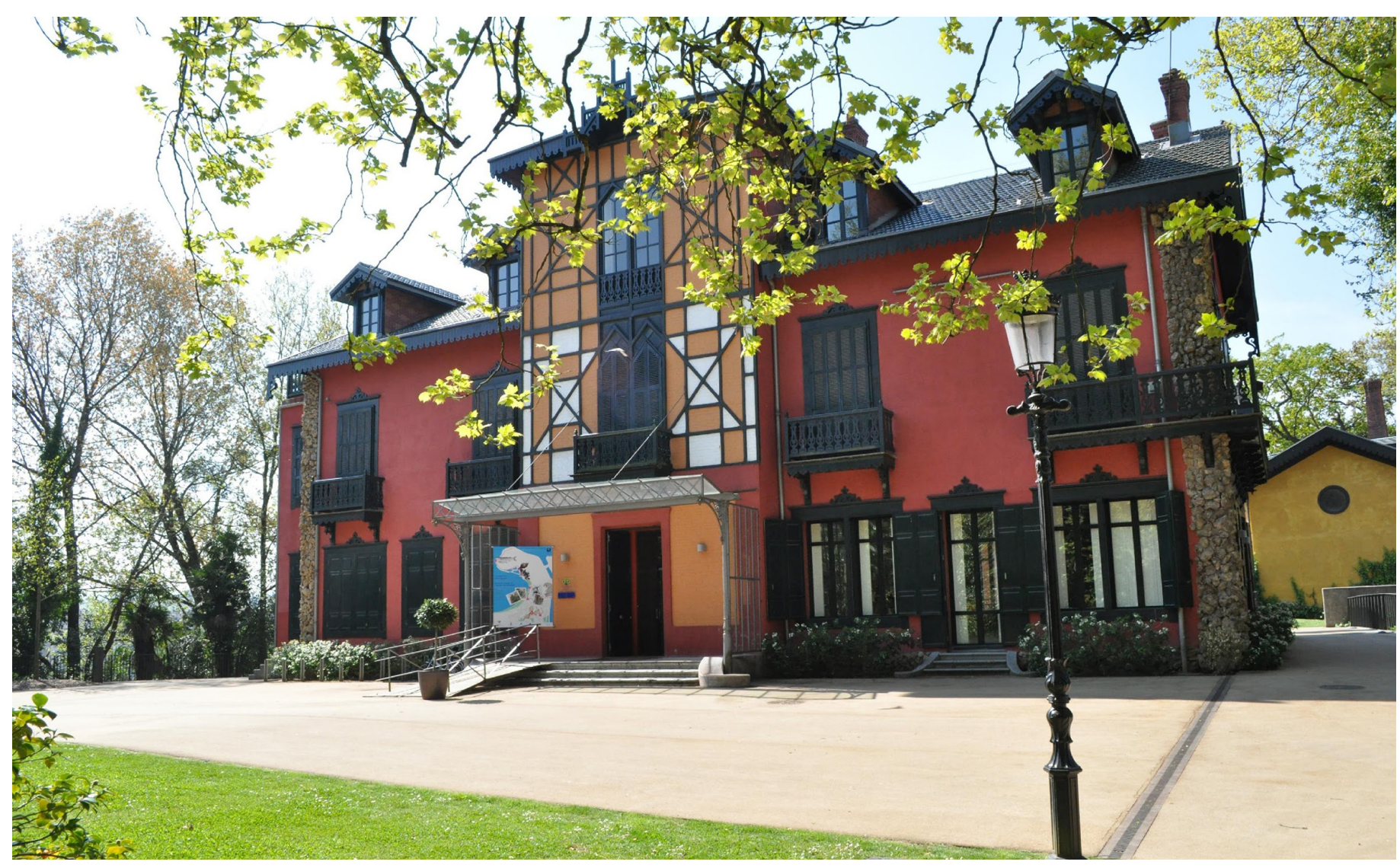

En este edificio se han adecuado dos espacios para desarrollar su actividad: uno que recoge el grueso de la documentación, para su análisis, clasificación y digitalización; y otro de $12 \mathrm{~m}^{2}$, situado en la biblioteca de Fundación Cristina Enea, donde se expone una selección de los documentos de mayor interés (Figura 3). Adosado a este espacio existe una zona de lectura y estudio.

En este equipamiento se pueden consultar libremente los materiales existentes, así como acceder a la base de datos de la Zikloteka mediante un ordenador que se pone a disposición del público.

Cuenta con atención personalizada, y está abierto al público de lunes a sábado, en horario de 09:30 a 13:30 h y de 15:00 a 20:00 h (en invierno, hasta las 19:00 h), y los domingos y festivos, de 10:00 a 13:30 h.

Por otra parte, se ha elaborado una herramienta informática para almacenar y gestionar toda la documentación recopilada.

La consulta de las bases documentales in situ, se ve complementada con la que se ofrece a través de un sitio $\mathrm{Web}^{3}$ diseñado para tal fin (Figura 4), dotado de un motor de búsqueda que dispone de dos opciones: una simple, en función de la autoría, título, ámbito territorial y/o temática; y una avanzada, que permite una búsqueda multicriterio a
Figura 2. Centro de Recursos Medio Ambientales. Fundación Cristina Enea. Fuente: Zikloteka. Centro de Documentación de la Bicicleta de Gipuzkoa. 
Figura 3. Espacio de consulta. Fuente: Zikloteka. Centro de Documentación de la Bicicleta de Gipuzkoa.

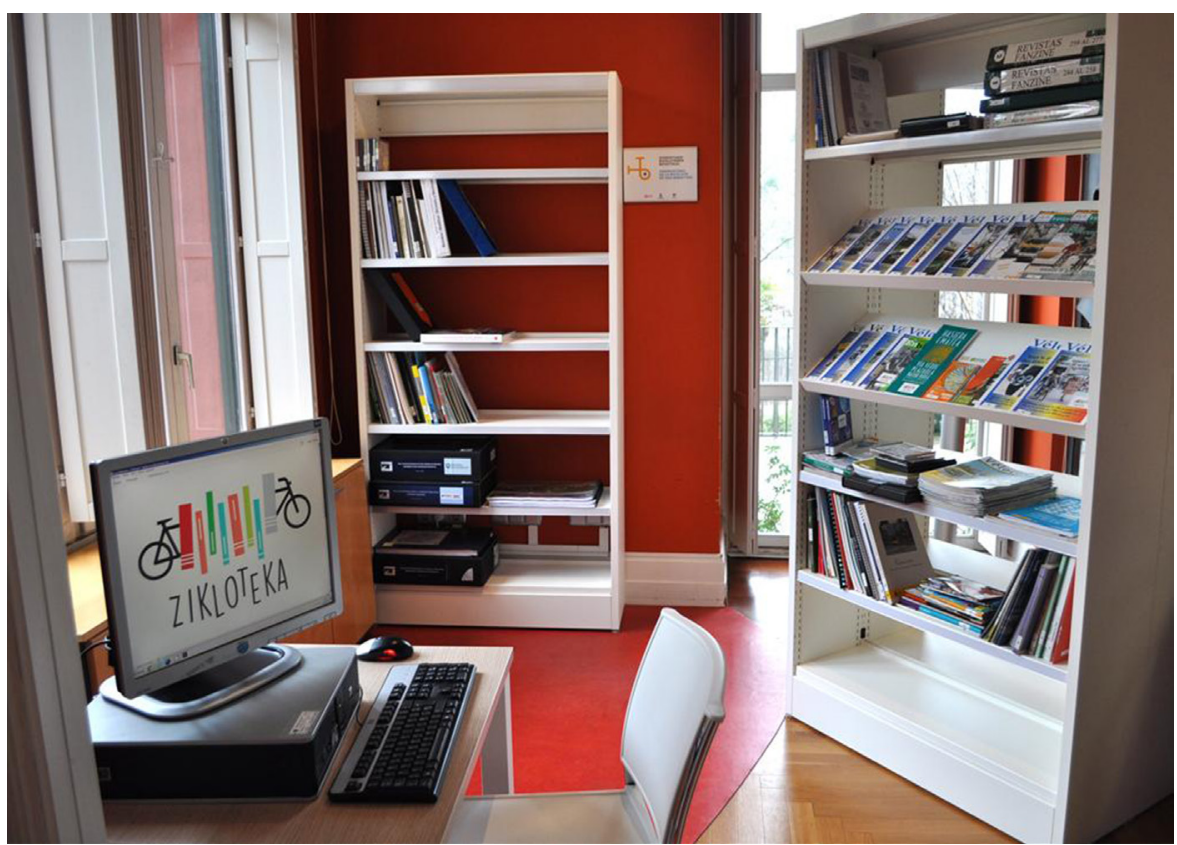

través de distintos campos, como el ISBN, el idioma, el año de edición, el formato o la fuente.

A través de estas búsquedas se le ofrece al usuario la posibilidad gratuita de descargar en línea la documentación digitalizada.

Como cabe imaginar, esta opción telemática es, en la práctica, considerablemente más utilizada que la presencial.

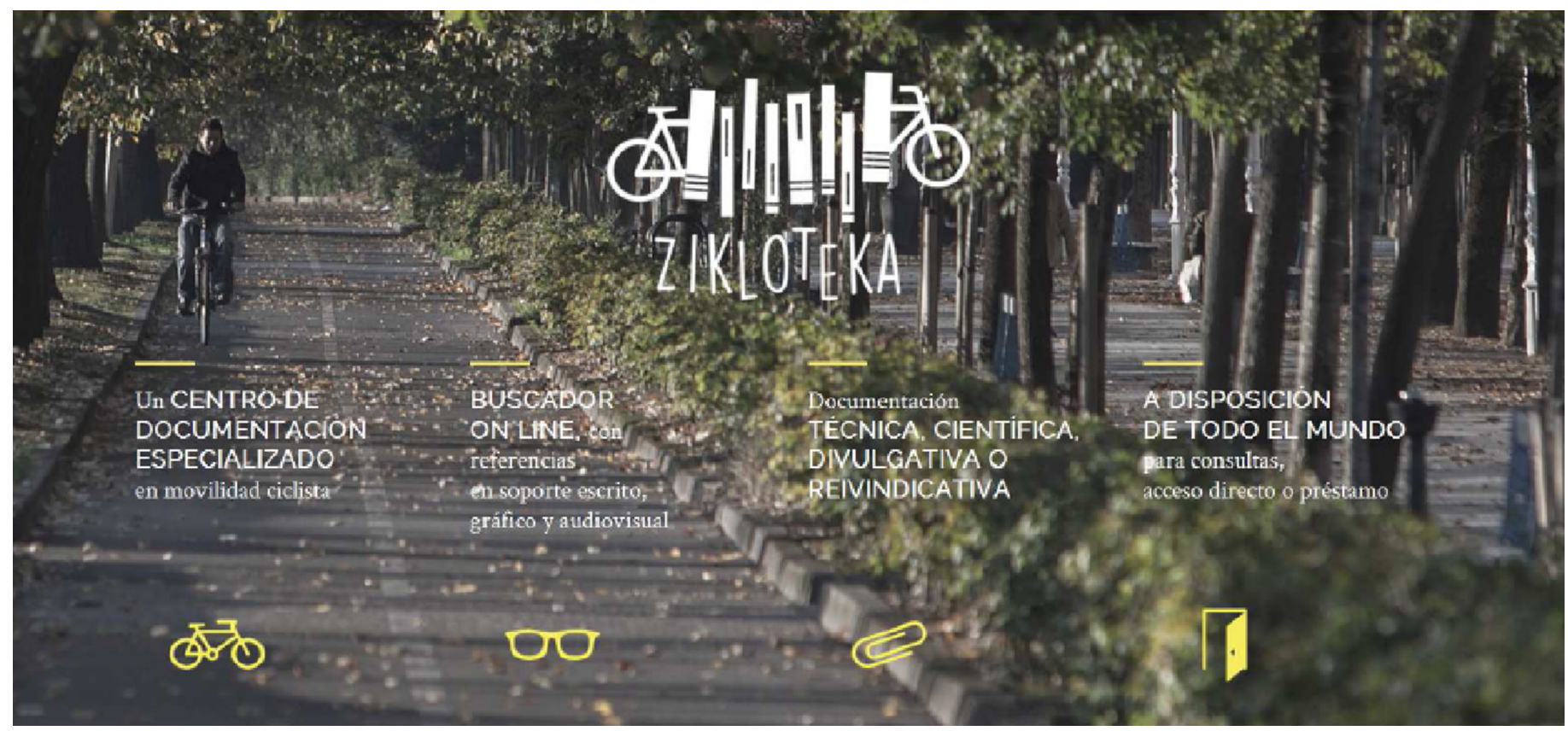

Figura 4. Sitio web de la Zikloteka (www.cristinaenea.eus/Zikloteka). Fuente: Zikloteka. Centro de Documentación de la Bicicleta de Gipuzkoa.
Otro servicio a destacar es el de préstamo gratuito de los fondos documentales físicos que están almacenados en sus dependencias.

Asimismo, es posible contactar con la Zikloteka a través del número de teléfono 943453 526, o del correo electrónico: zikloteka@donostia. eus.

A fin de ofrecer una magnitud del impacto generado, diremos que entre 2016 y 2019 la Zikloteka prestó servicio a más de 4200 usuarios, que bien han realizado una consulta presencial, visitado el sitio web o solicitado en préstamo algún documento. 


\section{Sistema de captación y clasificación de la documentación}

En una primera fase se estableció un sistema de seguimiento de la información relacionada con la movilidad ciclista urbana en Gipuzkoa, que aparece publicada en los medios de comunicación y redes sociales. Esta labor se ha complementado con un seguimiento de redes sociales y medios especializados, con el fin de captar documentación de interés. Esta información es clasificada semanalmente y se vuelca en un servidor para su puesta a disposición del público.

Por otra parte, se mantiene anualmente contacto directo con distintas entidades guipuzcoanas: ayuntamientos, mancomunidades, agencias de desarrollo, empresas del sector, asociaciones y federaciones ciclistas, universidades, etc., con el objetivo de solicitarles acceso a documentación relacionada de la que eventualmente puedan disponer.

En 2018, merced al Convenio de Colaboración suscrito con la Red de Ciudades por la Bicicleta, ${ }^{4}$ la Zikloteka afianzó más su reconocimiento de centro documental especializado al ampliar su ámbito territorial. De ese modo pasó de recopilar y difundir documentación relacionada con la movilidad ciclista exclusivamente de Gipuzkoa a incorporar aquella que pueden aportar las cerca de 1200 localidades representadas en esta Red.

En la actualidad sus fondos documentales cuentan con más de 3800 referencias, en soporte escrito, gráfico y audiovisual.

Se ha diseñado un sistema de voces y clasificación configurado y construido ex profeso, a fin de clasificar el material documental que se recaba, basado conceptualmente en los últimos avances sociales, técnicos y científicos en el ámbito de la movilidad sostenible.

Entendemos que este glosario, compuesto actualmente por $78 \mathrm{ca}-$ tegorías o etiquetas, constituye la principal aportación de la Zikloteka al área especializada en la documentación urbana y ambiental. ${ }^{5}$ Estas categorías estructuran un árbol que permite catalogar temáticamente cualquier información relacionada con la movilidad ciclista, y facilita al público la búsqueda documental en sus fondos.

\section{Difusión}

Con una frecuencia anual, mediante vía postal y electrónica se informa de la existencia de la Zikloteka a los ayuntamientos guipuzcoanos, a sus bibliotecas y servicios documentales, y también a otras instituciones y municipios de la Comunidad Autónoma del País Vasco, además de a todas las entidades socias de la Red de Ciudades por la Bicicleta.

Gracias a los acuerdos de colaboración suscritos con esta Red, así como con la coordinadora estatal de ciclistas urbanos ConBici ${ }^{6}$ y la revista de ciclismo urbano Ciclosfera, ${ }^{7}$ la Zikloteka cuenta con un banner de acceso a sus fondos en sus respectivos sitios Web y viceversa, lo que contribuye a difundir sus fondos ante un público más amplio.

Se mantiene, asimismo, relación con el Centro Nacional de Educación Ambiental, la Red de Centros de Información y Documentación Ambiental, el Centro de Educación e Investigación Didáctico-Ambiental (CEIDA) del Gobierno Vasco, y la plataforma de comunicación que el mismo tiene establecida con los centros escolares de la Comunidad Autónoma del País Vasco. Igualmente, se tiene contacto anual con las bibliotecas universitarias de la Universidad del País Vasco (UPV-EHU),
4 La Red de Ciudades por la Bicicleta es una asociación compuesta por 119 entidades locales (ciudades, mancomunidades, provincias y comunidades autónomas) que representan a 1194 municipios, que tiene por objeto la generación de una dinámica entre las ciudades españolas con el fin de facilitar, hacer más segura y desarrollar la circulación de los ciclistas, especialmente en el medio urbano. http://www.gipuzkoabizikletaz.eus/es/-/ ziklotekaren-eta-red-de-ciudades-por-labicicleta-erakundearen-arteko-lankidetzaakordioa-sinatu-du-aldundiak.

5 Ver anexo.

6 ConBici es una coordinadora de ámbito estatal que agrupa a 63 organizaciones locales y provinciales de ciclistas urbanos. https://conbici.org/noticias/ noticias-nacionales/zikloteka-y-conbiciacuerdan-colaborar-para-impulsar-la-documentacion-sobre-movilidad-ciclista. 7 Ciclosfera es la única revista española centrada específicamente en el ciclismo urbano. 


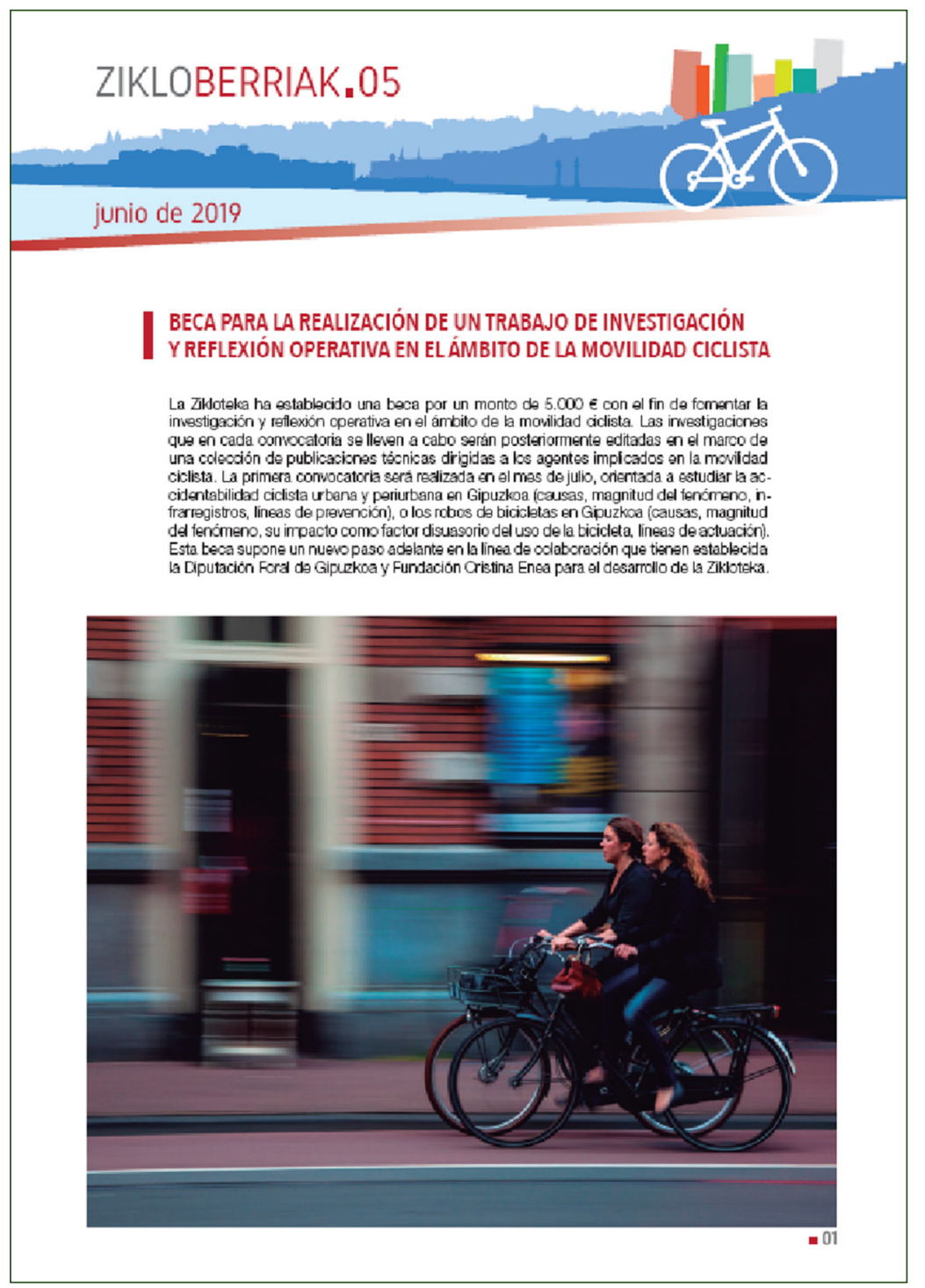

de la Universidad de Deusto y de TECNUN (Universidad de Navarra), para la difusión del servicio entre el alumnado y el profesorado.

Para apoyar la difusión de sus prestaciones, Zikloteka edita anualmente un tríptico bilingüe donde se explican sus objetivos, servicios y funcionamiento, y que se distribuye en casas de cultura, bibliotecas públicas y universitarias, servicios de urbanismo y de movilidad municipal, centros escolares y entidades deportivas.

A partir de 2017 publica con periodicidad semestral el boletín electrónico Zikloberriak que da a conocer las principales novedades recopiladas y una selección documental, así como noticias o convocatorias relacionadas con el fomento y el uso cotidiano de la bicicleta (Figura 5).

Hasta diciembre de 2019 se han editado 6 números que se distribuyen directamente a 520 suscriptores. Fruto de la colaboración establecida con la revista Ciclosfera y la coordinadora ConBici, estas entidades contribuyen también a la selección de documentos que consideran de especial interés y que se referencian de manera destacada en cada boletín.

Para poner en valor y dar a conocer la existencia de la Zikloteka y de los servicios que presta, se procura estar presente en los principales eventos sobre la movilidad ciclista $\mathrm{u}$ otros ámbitos relacionados. Así se ha partici-

Figura 5. Carátula del boletín electrónico Zikloberriak. Fuente: Zikloteka. Centro de documentación de la Bicicleta de Gipuzkoa pado en el Congreso Nacional de Medio Ambiente (CONAMA 2016, Madrid); en el XIV y XV Congreso Ibérico "La Bicicleta y La Ciudad" (Zaragoza, 2017; Valencia, 2018); en el XVII Seminario de Centros de Documentación Ambiental y Espacios Naturales Protegidos (Zaragoza, 2018); y en el encuentro estatal de ConBici (San Sebastián, 2018).

Por otra parte, la Zikloteka se ha incorporado al directorio de la Red de Centros de Documentación Ambiental (RECIDA), impulsada desde el Centro Nacional de Educación Ambiental (CENEAM), del Ministerio para la Transición Ecológica; y al Seminario de Centros de Documentación Ambiental, dependiente de esta misma Red.

\section{Dirección y gestión del centro documental}

La dirección y coordinación técnica del centro es compartida entre la Diputación Foral de Gipuzkoa y Fundación Cristina Enea, bicefalia institucional e intelectual del proyecto.

Fundación Cristina Enea asume la gestión de la Zikloteka, y asimismo:

- Es la depositaria y custodia de los archivos que se generan y de sus fondos y materiales. 
- Proporciona soporte humano, físico e informático.

- Se responsabiliza de la coordinación y relación con los proveedores de información y entes colaboradores; de la alimentación y gestión del sitio Web; así como de la selección y clasificación documental.

Por su parte, el Departamento de Movilidad y Ordenación del Territorio de la Diputación Foral de Gipuzkoa sufraga el gasto relativo a la adquisición de fondos documentales y otros bienes y servicios relacionados con la gestión y promoción del centro que, si bien son de su propiedad, son custodiados por Fundación Cristina Enea.

Con el objetivo de poder realizar una evaluación anual de la gestión de la Zikloteka y un seguimiento histórico de su evolución, se ha establecido un sistema de indicadores cuya implementación se inició en 2016.

A partir de ese sistema de indicadores se elabora una memoria anual, que recoge también los principales retos para la gestión del centro que se plantean para el año próximo.

\section{Línea de investigación y colección de publicaciones técnicas}

En 2020 la Diputación Foral de Gipuzkoa y Fundación Cristina Enea decidieron implantar un nuevo eje de trabajo, tras estimar ya consolidados los sistemas de gestión y colaboración establecidos, y haberse logrado ampliar sustancialmente su ámbito territorial.

Se trata de un eje de trabajo consistente en la puesta en marcha de una línea de investigación a través de una convocatoria anual de una beca, que se realizará por primera vez a principios de 2021, para la realización de proyectos de investigación en el ámbito de la movilidad ciclista. De este modo se desea contribuir a incrementar el aún modesto número de investigaciones y reflexiones operativas que se llevan a cabo en torno a las diferentes vertientes de la movilidad ciclista, a fin de que redunden posteriormente en una mejora del conocimiento técnico de los agentes públicos, privados y ciudadanos que intervienen en la misma.

Para contribuir a este fin, esta iniciativa vendrá acompañada del lanzamiento de una colección de publicaciones técnicas, que tendrá como función editar las investigaciones que se hayan becado y, eventualmente, otros estudios, informes o ensayos relacionados con este área.

\section{Balance valorativo}

Tras haber transcurrido 4 años desde que se inició la andadura de la Zikloteka, nos atrevemos a afirmar que se ha configurado un centro público documental especializado en la movilidad ciclista, que es referencial y único en Europa.

Se trata de un recurso valioso para la promoción de la movilidad ciclista, ya que recopila y gestiona la producción administrativa, científica, técnica y ciudadana que se genera en este ámbito, y la pone gratuitamente a disposición de los sectores profesionales y ciudadanos interesados, que así se pueden beneficiar del conocimiento colectivo generado. 
Consideramos que cabe concluir una valoración positiva de su aún breve, pero intensa trayectoria, si atendemos a los resultados obtenidos:

a) Se ha logrado consolidar sus sistemas de gestión y colaboración con los principales agentes institucionales y asociativos del ámbito de la movilidad ciclista.

b) Se ha ampliado sustancialmente su ámbito territorial, partiendo del territorio de Gipuzkoa a las cerca de 1200 administraciones públicas representadas en la Red de Ciudades por la Bicicleta.

c) Continúan en progresivo aumento tanto sus fondos documentales (3800 registros) como el número de personas usuarias (más de 4200 usuarios entre 2016 y 2019) y suscriptoras del boletín electrónico (520).

Como cabe suponer, la Zikloteka lejos de adoptar una actitud complaciente ante estos resultados, aspira a poder alcanzar en el medio plazo unos niveles de difusión y utilización sensiblemente superiores a los actuales. Los avances hacia este objetivo estarán condicionados por la dotación de recursos económicos que se asignen, pero también por el ritmo al que el ámbito de la movilidad ciclista va adquiriendo un estatus normalizado en las esferas administrativas, profesionales, académicas y ciudadanas.

Confiamos, pues, en que en un horizonte cercano el mayor impacto de la Zikloteka sea efectivamente indicador del avance en la normalización de la movilidad ciclista, tanto en el conjunto de nuestra sociedad, como en el seno de las administraciones públicas. 


\section{Referencias bibliográficas}

Zikloteka (2017-2019). Colección de boletines electrónicos «Zikloberriak» (números del $1 .^{\circ}$ al $6 .^{\circ}$ ). Recuperado el 23 de marzo de 2020 de: http:/ / www.cristinaenea.eus/zikloteka/es/hasiera.

Bergua Jiménez, Edorta y González Baragaña, Manu (2020). Zikloteka, centro de documentación de la bicicleta. Una iniciativa avanzada de recopilación, tratamiento y difusión de documentación sobre la movilidad ciclista. Hábitat y Sociedad, 13, 265273.

<http://dx.doi.org/10.12795/HabitatySociedad.2020.i13.15>

$\bigcirc$ 


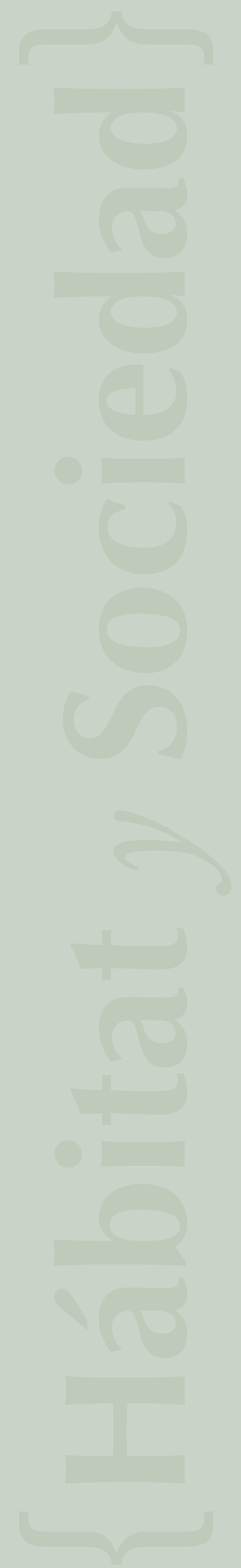

